\title{
OP 012 THE USE AND CORRELATION OF PATIENT REPORTED OUTCOME MEASURES (PROMS) IN A HOSPITAL OUTPATIENT SETTING
}

Clare Farrington, ${ }^{1,3}$ Bill Noble ${ }^{2}$. 'Sheffield Teaching Hospitals Foundation Trust, Sheffield, UK; ${ }^{2}$ Academic Unit of Supportive and Palliative Care, University of Sheffield, UK; ${ }^{3}$ University of Cardiff, UK

10.1136/bmjspcare-2014-000654.12

Background Consultation And Relational Empathy (CARE) and Patient Enablement Instrument (PEI) PROMs were highlighted by a task group of the association of palliative care as useful research tools for assessment of the consultation process.

Aims Assess the feasibility and acceptability of using CARE and PEI PROMs in a hospital outpatient setting. Also the correlation between these PROMs.

Methods Set within a feasibility study to examine the effect of Advanced Communication Skills Training ACST on PROMs. Two data collections of 75 questionnaires posted to sequential outpatients following a consultation with a participating doctor. Data analysed using descriptive statistics and Spearman rank correlation to test PROMs association.

Results Patients returned over half the questionnaires (802, $53.5 \%$ ). Respondents were representative of outpatient population with regard age and gender. Majority of questionnaires were evaluable: CARE 801 (99.9\%), PEI 712 (88.8\%). Spearman's rank correlation coefficient $(r=0.33 \quad \mathrm{p}=0.01)$ showed a moderate positive correlation between CARE measure and PEI score in hospital outpatients. The proportion of consultations that were defined as enabling (PEI score six or more) tended to increase, and those with poor enablement (PEI score two or less) decrease, as CARE measure score increased. No full enablement occurred (PEI maximum score $=12$ ) when CARE score was 30 or less (CARE measure items averaging poorgood). Enablement occurred most frequently (52.3\%) when a maximum CARE measure score (50) was assigned.

Conclusions The postal method of administering these PROMs appears a feasible and acceptable method to assess hospital outpatient consultations. As patients' rating of their doctors communication skills and empathy (CARE scores) increase the tendency is for greater patient enablement (PEI scores) though not uniformly. Not all consultations where doctors were scored as having excellent communication skills and empathy were enabling. This indicates that empathy is a necessary but not sufficient condition for enablement. 